\title{
Bioanalytical method validation guidance language and a decade of progress
}

\author{
Stephen Lowes*,1 \& Michael Brown ${ }^{1}$ \\ ${ }^{1}$ Bioanalytical \& ADME Laboratories, Q Squared Solutions, 19 Brown Road, Ithaca, NY 14850, USA \\ *Author for correspondence: Stephen.Lowes@q2labsolutions.com
}

\begin{abstract}
' 10 years ago, bioanalysts were operating to a single BMV Guidance (US FDA) but the scene was set for a modernization of regulatory language that would reflect the ongoing practice of many bioanalytical laboratories."
\end{abstract}

First draft submitted: 18 February 2019; Accepted for publication: 5 March 2019; Published online: 15 April 2019

Keywords: $\mathrm{BMV} \bullet$ harmonization $\bullet$ regulations

Over the last 10 years, bioanalytical method validation (BMV) has evolved in terms of science and health authority guideline language. Bioanalysts in 2019 need to be cognizant of a multitude of guidance and the associated interpretations around the globe. The transgression from a single regulatory document in effect in 2009, to today's status has been documented through many publications in Bioanalysis. As such, this reflection paper recalls some of the pivotal points that have impacted regulated bioanalysis and what may lie ahead. At the time of writing, a single harmonized global BMV guideline is proposed and while appealing in concept, the complexity and diversity of bioanalytical approaches challenges any detailed prescriptive instructions on how to validate all assays. However, the fundamental attributes of a BMV that generate trustworthy data, have sustained the last decade and we can expect them to serve us well into the future. The authors reflect on key regulatory language developments and consider the lessons served as we take the bioanalytical discipline forward.

It is interesting to track back through the volumes of the Bioanalysis journal to see the frequency of reference to regulations and BMV Guidance. There, it is in Bioanalysis Volume 1 issue 1 (April 2009); the conference report on the second Calibration and Validation Group workshop held the previous year in Toronto, Canada [1]. The report outlines where we were a decade ago in discussing and debating current topics around BMV regulations. Of course, along with the Calibration and Validation Group workshop evolving into the Workshop on Recent Issues in Bioanalysis, the regulatory aspects of bioanalysis have also developed in coverage and complexity. At least, it seems that way. Fundamentally, however, we are still tasked with demonstrating the performance of a bioanalytical method against the criteria pillars of accuracy, precision, sensitivity, selectivity, stability and reproducibility, as we were in 2009 (and before). Considering what has changed and what remains consistent over the last decade, this reflection paper summarizes some of the key milestones that stand out to the authors and speculates what may lay ahead.

10 years ago, bioanalysts were operating to a single BMV Guidance (US FDA) [2] but the scene was set for a modernization of regulatory language that would reflect the ongoing practice of many bioanalytical laboratories. Pivotal White Papers that accommodated the expectations from the early Crystal City workshops and from the ligand-binding assay (LBA) community were considered essential complements to the 2001 BMV Guidance [3-6]. Then came the EMA contribution to BMV regulatory guidance (effective in 2012) [7] and the ensuing debates around harmonization began. That discussion has not stopped to this day. With several other global health authorities issuing BMV Guidance, which largely replicated EMA, an inordinate amount of workshop and conference sessions would focus on any actual or interpreted differences. The FDA issuance of their updated BMV Guidance in draft form in 2013 [8] added further debate and introduced the most significant changes, since the 2012 EMA BMV Guidance. However, the US FDA document remained in draft until last year (2018), leaving some degree of ambiguity and confusion. With finalization of the FDA 2018 BMV Guidance [9], a new round of debate has begun against the background appeal for BMV harmonization and consistency of scope. 
As writing this reflection paper, another BMV Guidance intended to bring harmonization is now anticipated. The International Council for Harmonisation of Technical Requirements for Pharmaceuticals for Human Use $(\mathrm{ICH})$ has recently released a draft BMV Guidance under the M10 initiative. The ICH M10 process involves the multitude of global health authorities with the intent of addressing the BMV harmonization challenge. No doubt, the resulting finalized document will garner appropriate attention and hopefully will become the de facto reference for bioanalysts.

In parallel to the last decade of evolution of guidance language, there have been changes in what bioanalysts are tasked with doing. The rise of biologics is probably the most significant development. The development of biotherapeutics at the time of the first guidance was in its relative infancy and the recommendations often favored language and approaches more relevant to chemical entities and, in fact, suggested LBAs be compared with a validated reference method. An engaged community put forward a series of publications that provided granularity to the best practices for validation of macromolecule quantitative assays with a focus on nonlinear curve fit model selection, approaches to controlling critical reagents and assessment of selectivity and specificity, for instance $[3,10,11]$. Technology and technique used by the bioanalysts to address new analyte modalities have also changed considerably over the last 10 years. We have seen this challenge the guidance language of the past with the likes of dried blood spot (DBS) and other bioanalytical tool developments. In addition, the pace of innovation with respect to new therapeutic modalities such as cell and gene therapies and sophisticated viral capsid delivery of small-molecule payloads will demand transformative thinking with the bioanalyst. The authors expect increasing challenges in accommodating the opportunities of technology development with any detailed prescriptive BMV Guidance language but, perhaps, the historical lessons can help us going forward.

\section{Year 2009 (the launch of Bioanalysis: the Journal)}

In 2009, bioanalysts supporting primarily pharmacokinetic (PK) studies with data intended for regulatory submissions were very familiar with the FDA 2001 BMV Guidance. We were also familiar with some of the shortfalls of that document. Two Crystal City meetings in 2005 and 2006 (co-organized with the American Association of Pharmaceutical Scientists [AAPS]) had established the FDA's expectations for demonstrating bioanalytical method performance above and beyond the 2001 Guidance. In particular, the need to demonstrate incurred sample reanalysis (ISR) had been established and the workshop reports of Crystal City III [3] and IV [4] became integral to bioanalytical laboratory standard operating procedures (SOPs). ISR has evolved to the present day as a requirement, which had a contentious beginning but is now generally accepted as a valuable assessment of assay reproducibility. That is not to say every assay and every bioanalytical need is dependent on ISR data to enable decisions. That was not true in 2009, and it is not true today. For instance, there is a lively and warranted discussion ongoing around the value of the ISR experiment for biomarker assays when endogenous quality control samples (QCs) are tracked across bioanalytical batches and runs. However, the authors raise this point here as an example of bioanalytical regulation development from 10 years ago that has meaningful relevance to today's challenges.

While the chromatographic bioanalysts wrestled with ISR, it is fair to say that the ligand-binding bioanalytical community had minimal assistance from regulatory guidance language in 2009. LBAs relied more on key reference papers from opinion leaders and consortia. Several highly regarded White Papers directly addressed LBA method development and validation $[5,6,12]$. The influence of these papers even on today's practices should not be underestimated.

Technology developments in the bioanalytical lab have largely followed an iterative path since LC-MS displaced LC-UV in the 1990s. Certainly advances in sample preparation efficiencies, chromatography separations and MS sensitivity can be referenced. Arguably, more significant technology arrivals can be cited in the ligand-binding field during the early 2000s including electrochemiluminescence (e.g., Meso Scale Discovery-ECL [MSD]), chip-based technologies and advanced microfluidics (e.g. surface plasmon resonance [SPR] and Gyrolab). However, in 2009 the technique of DBS sample collection and subsequent analysis garnered most discussion in regulated bioanalysis. The regulatory aspects of this technique were vigorously debated alongside the science. The advances of microsampling and the three Rs (reduce, refine, replace) over conventional sample collection continue to this day, although some of the purported limitations of DBS have precluded its widespread adoption. Regulatory language from the FDA continues to reference DBS approaches [9] and echo some of the discussions from the 2009 period serving as an example of what bioanalysts need to consider when new technology opportunities present. 


\section{Period 2009-2012 (Bioanalysis: the Journal becomes a mainstay)}

The 4-year period of 2009-2012 ushered in some significant regulatory developments for bioanalysts. The drafting of the EU's EMA BMV Guidance became evident in 2009 and ultimately resulted in its release in 2011 (effective February 2012) [7]. This development induced open discussions and collaborative industry response on a scale previously not recognized. The EMA guidance itself was a welcome update of regulatory language that reflected ongoing practice of the time. As such, bioanalytical laboratories were largely already in compliance, however, the fact that there were now two effective BMV Guidance documents addressing a global industry raised the concern over harmonization. This was somewhat warranted with Brazil ANVISA releasing Resolution RDC 27 in 2012 [13], covering minimal requirements for BMV for studies intended for submission and evidence Japan's Ministry of Health Labor and Welfare was drafting its own BMV Guidance too [14,15]. The response from industry was to escalate dialogue with regulatory bodies and organize efforts to harmonize over regulated bioanalytical practices. In 2010, industry scientists from EU, the USA and Canada formed the Global Bioanalysis Consortium (GBC). Other regional groups of bioanalysts from around the world, including teams from Brazil, India, China and Japan subsequently joined the GBC nonprofit volunteer initiative. With a mission to recommend harmonized BMV language, the GBC organized into subgroups around pivotal areas of BMV practice and technique. The outcome was a series of consensus papers reflecting chromatographic and LBA validation strategies that regulatory language can be based upon [16]. Today's bioanalyst is advised to review these papers and articles from the work of GBC teams as they are still valuable and relevant to ongoing discussions around regulatory language development.

Another regulatory development is worth mentioning from this period that has, to this day, significant influence on SOPs of bioanalytical laboratories. In 2012, the EMA issued their reflection paper for laboratories performing analysis of samples supporting clinical trials [17]. This heralded the confluence of GLP and GCP into the logical, good clinical laboratory practices. The reflection paper of good clinical laboratory practices addressed the need of bioanalysts supporting regulated clinical studies to be compliant with patient confidentiality and safety along with the documenting standards of GLP. In practice, this was already an expectation of bioanalytical laboratories, nonetheless from 2012 it became a requirement worth noting under EMA regulations.

Paralleling the PK bioanalysis discussions was another branch of drug development bioanalytical science that gained increased regulatory attention in this time period. In 2009, the Guidance for Industry: Assay Development for Immunogenicity Testing of Therapeutic Proteins was published in draft by the FDA and has now been finalized [18]. This followed the earlier EMA Guideline on Immunogenicity from 2008, but brought into full focus the bioanalytical requirements to investigate antidrug antibodies (ADA). Immunogenicity assessment has become a key pillar in the evaluation of safety and efficacy of drugs in development. ADA assays are designed to detect antibodies that could mediate unwanted biological or physiological consequences such as neutralizing activity or hypersensitivity responses. There are well-documented cases of life-costing responses with recombinant erythropoetin (EPO), megakaryocyte growth and development factor (MGDF) and thrombopoietin (TPO) as well as less devastating but impactful effects of immune responses to biotherapeutics [19-21].

Over the last 10 years, the field of immunogenicity has matured but finds itself in a similar position to where PK validation was in 2001, with a broad approach to testing. Over this period, there has also been a convergence of data integration between the fields of biologic quantitation and the immune response to these biotherapeutics. The FDA expects that "The assays should have sufficient sensitivity to enable detection of ADA before they reach levels that can be associated with altered pharmacokinetic (PK), pharmacodynamic (PD), safety or efficacy profiles" [18]. The recently finalized FDA immunogenicity guidance adopts the EMA integrated summary of immunogenicity report [22], where including the results of linear or nonlinear correlation analyses between ADA status, and titers with PK, pharmacodynamic, efficacy and safety data. Another area of confluence was the recognition by the FDA that data generated from appropriately designed and sensitive PD marker and/or PK assays that provide insight to clinical activity may supplant the necessity of a standalone ADA neutralization assay.

\section{Period 2012-2017 (impact of a draft BMV guidance)}

A response from FDA to the EMA 2012 BMV Guidance was expected and it arrived as a draft in mid-2013 [8]. Consistency with the EMA language was anticipated but some significant deviations and additions from past guidance were introduced by the FDA. Debate about the draft guidance was immediate and commenced with the Crystal City V meeting and then a historically high number of written responses to the agency. It is fair to say that bioanalytical scientists had issues with multiple aspects and wrestled with interpretation of the ambiguously, and at time contentiously, written document. Confusion over ISR criteria, inclusion of failed runs statistics, design of 
some stability validation assessments, addressing biomarkers and DBS assays generated considerable debate in the bioanalytical community.

Despite the FDA stating on several occasions that the 2013 BMV Guidance was 'a draft' and should not be implemented, reports started to emerge that regulatory inspectors were referring to specific aspects of the draft. This continued over 5 years with bioanalysts exchanging experiences and opinions at national and international conferences, workshops and meetings on the topic. Consequently, most bioanalytical laboratories retained use of the EMA 2012 Guidance for SOPs and general practice but the FDA 2013 Draft BMV language always loomed in the background until recently.

One of the more significant additions to BMV Guidance introduced by the FDA with the 2013 draft was the concept that biomarkers assays may be considered within scope. The Crystal City V workshop and the subsequent report [11] did not sufficiently address the recommendations on biomarkers and endogenous compounds thus leading to Crystal City VI meeting in 2015. Here, chromatographic (i.e., LC-MS) and LBA scientists from both the traditional bioanalytical (i.e., PK supporting) and diagnostic clinical chemistry laboratories joined the discussion. The discussion was either a reminder or an education on the established practice and regulatory science associated with biomarker assays. Biomarker assays supporting clinical assessments already have extensive precedence with Clinical \& Laboratory Standards Institute guidelines, Clinical Laboratory Improvement Amendments certification and College of American Pathologists accreditation predominating. Even so, the FDA has maintained a position that biomarker bioanalytical data used for safety, efficacy and product labeling submissions need to be fully validated, that is, to BMV standards. Currently, it is clear that when biomarker data is used to support a regulatory decision-making, such as the pivotal determination of safety/effectiveness or label instructions, the assay should be fully validated consistent with the scientific principles in the BMV as applies to drug product. However, with the diversification of the therapeutic modalities and the increase in the number of clinically validated biomarkers that may not be amenable to support in a traditional bioanalytical laboratory, consensus was lacking. A working group, FDA/C$\mathrm{PATH}$, has been working on a detailed and definitive recommendation for biomarker method validation; Scientific and Regulatory Considerations for the Analytical Validation of Assays Used in the Qualification of Biomarkers in Biological Matrices [23] that promises to help clarify appropriate validation activities in a fit-for-purpose (FFP) manner.

Related to this is the best practice for supporting testing of biotherapeutics that are endogenous compounds and biomarkers. There is a growing but not completely formed agreement about the design of calibration curves and QC samples. One area of that could use clarification is around the use of a stripped biological matrix to prepare calibration standards versus a defined buffer that exhibits parallelism with matrix-based samples. Other areas include how to account for the endogenous concentrations in matrix, the relevance of recovery of overspiked exogenous surrogate calibrator, bridging calibrator changes and recognizing the limitations and risks of comparing endogenous analyte concentrations across methods for assays that are not definitive quantitative assays (e.g., testosterone). Across the bioanalysis community, the debate around regulated bioanalysis of endogenous compounds and biomarkers is expected to continue.

The biomarker discussions have raised a fundamental challenge with BMV Guidance. That is, the prescriptive language that has arguably served us well for bioanalytical studies supporting PK of xenobiotic drugs does not apply as well for endogenous analytes. This is particularly true for analytes where the reference standard is essentially a surrogate for the analyte of interest. Recombinant reference standards of protein biomarkers may be the best we can practically access but any differences may have a systematic bias on accuracy. Assay validation experiment criteria need to be considerate of such inherent factors. If we are to accommodate the new challenges presented by drug development, biology and disease treatment, then we must be able to adapt our bioanalytical practices to the current objectives and purpose of the measurements. FFP strategies are most likely to help in the quest for efficient bioanalysis and progress that meets the demand of modern medicine.

There will be difficulties with implementing FFP as it challenges the history of BMV. Concern is warranted based upon even recent experience. An example of regulatory interpretations that diverged from established practice happened in 2015 and resulted in the industry changing to accommodate a health authority perspective. Health Canada issued a notice that required stability testing to employ three individual samples derived from separate containers. Previously, common practice in bioanalytical laboratories was to take three aliquots from the same container - a significantly more efficient and less expensive approach. Of course, the potential for stability differences between three separate containers cannot be exhaustively disproved, but neither was there a weight of evidence that suggests there was a concern with the common practice of a single container assayed with three 
replicates. This example of regulatory influence needs to be recognized, however, bioanalytical science is supported by a weight of statistics and, as such, risk-based decisions should still dominate. If FFP is to further bioanalytical practice, then the onus will be with the bioanalyst to defend the science conducted with documented data that substantiates the approach taken to validate an assay.

\section{7-2019: current state of bioanalysis}

By 2017, there were at least six health authority guidance documents around the globe that directly influenced bioanalysis. Adding to the USA, EMA, ANVISA and Japan (two guidance: small molecule and large molecule), the China CFDA proposed their own BMV Guideline (English translation pending). In May 2018, the FDA unexpectedly finalized and released for use the update to the 2013 draft [9]. Significant improvements in the text were noted around readability and adoption of recommendations from industry. Largely consistent with current practice, the release has resulted in minimal changes to most bioanalytical laboratory SOPs regarding PKsupporting assays. The summary of assay validation criteria (differentiated by chromatographic assays and LBA) in a convenient appendix table format has been well received by the bioanalytical community. Consistency with EMA Guidance around ISR and assay performance criteria for accuracy, precision, sensitivity, selectivity and stability is also noted. The FDA 2018 BMV Guidance also emphasized method development and ultimately the importance of demonstrating an assay is suitable for the intended use. The authors of this paper interpret such language as helping future proof the Guidance to new technologies and bioanalytical practice, which is likely to accompany progress in therapies and disease management.

There are some nuances to the FDA 2018 BMV that the bioanalytical community are engaging with the agency at the time of writing this paper. The influence of reference compound retest dates on solution expiration as well as appropriate accuracy and precision statistics are focal points but are expected to be resolved. While assay validation stability experiments are largely consistent with common practice, the reference to comedication influence on analyte stability has drawn some critique. These finer points of the FDA Guidance may be addressed with the ICH M10 initiative.

Out of the multitude of BMV Guidance emerging around the globe came the interest of the ICH. In 2016, $\mathrm{ICH}$ endorsed the concept of a multidisciplinary guideline on bioanalytical methods and their use for the analysis of nonclinical and clinical samples [24]. Consistent with the mission of $\mathrm{ICH}$, the plan of reaching a harmonized guidance to supersede regional BMV documents and thus 'promote prompt, rationale and effective nonclinical and clinical studies' was initiated. An expert working group comprising nominated chromatography and ligand-binding bioanalysts from the ICH member countries and observers was established to execute the M10 BMV business plan. A key element of the plan was to derive a BMV Guidance that all regional health authorities would defer to as the definitive Guidance. As of the writing of this article the draft M10 Guidance had just been released. In 2019, there will be opportunity for public comment on the draft document and possible finalization soon thereafter. Since the founding of the GBC in 2010 (see earlier [16]), the objective of a single global BMV Guidance has been desired. We can anticipate individual and even regional interpretation of such a guidance may vary but nonetheless reference to one document should be an improvement over today's multiguidance status.

\section{Conclusion \& future of bioanalysis regulations}

The last 10 years of bioanalysis have been influenced by regulatory guidance and associated inspection interpretation. Various industry consortia leveraging online and in-person communication channels have become adept with exchanging information on the FDA Form 483s and equivalent observation citations. In parallel to regulatory language technology developments, drug modalities and targeted analytes have all influenced change in the practice and procedures of the modern bioanalyst. We can expect this to continue and evolve further as the demands on bioanalytical labs to adapt to developments in disease treatment arise. While harmonized BMV guidance may currently be a realistic expectation, the bioanalyst tasked with quantitative determination of biomarkers, immunogenicity profiling or accommodating a new generation of bioanalytical technologies will not be able to rely on any prescriptive guidance language for all situations encountered. Whether outsourcing bioanalysis to a contract research organization $(\mathrm{CRO})$ or conducting the work within the sponsors' own laboratories, bioanalytical skill set and experience will continue to be a strong influence on achieving the desired outcome. While it is exciting to learn about genomic and immunotherapy approaches to fighting disease, these and other fundamental developments in furthering medicine will likely challenge the established models of bringing safe and effective pharmaceuticals to market. Bioanalysis will undoubtedly be called on to adapt to support such progress. 
Against the backdrop of the last 10 years of bioanalytical procedure and practice development has been an iterative evolution of regulatory language. How bioanalysis regulations will track the needs and expectations over the next decade is intriguing to contemplate. Lessons from the past would suggest that bioanalytical guidance forms a useful basis for compliance and relative consistency. The challenge going forward will be to accommodate an escalating pace of technology developments and the growing scope of bioanalytical laboratories. The authors hope that reflections such as this paper will help us recognize what can continue to serve bioanalysts well but also acknowledge where new and additional protocol needs to be established to enable the next generation(s) of our discipline.

\section{Financial \& competing interests disclosure}

The authors have no relevant affiliations or financial involvement with any organization or entity with a financial interest in or financial conflict with the subject matter or materials discussed in the manuscript. This includes employment, consultancies, honoraria, stock ownership or options, expert testimony, grants or patents received or pending, or royalties.

No writing assistance was utilized in the production of this manuscript.

\section{References}

1 Savoie N, Booth BP, Bradley T et al. 2008 White paper: the 2nd Calibration and Validation Group Workshop on recent issues in good laboratory practice bioanalysis. Bioanalysis 1(1), 19-30 (2009).

2 US FDA. Guidance for Industry: Bioanalytical Method Validation (2001).

3 Viswanathan CT, Bansal S, Booth B et al. Quantitative bioanalytical methods validation and implementation: best practices for chromatographic and ligand binding assays. Workshop/Conference Report. AAPS J. 9(1), E30-E42 (2007).

4 Fast D, Kelley M, Viswanathan CT et al. Workshop report and follow-up: AAPS workshop on current topics in GLP bioanalysis; assay reproducibility for incurred samples - implications of Crystal City recommendations. AAPS J. 11(2), 238-241 (2009).

5 DeSilva B, Smith W, Weiner R et al. Recommendations for the bioanalytical method validation of ligand-binding assays to support pharmacokinetic assessments of macromolecules. Pharm. Res. 20, 1885-1900 (2003).

6 Smolec J, DeSilva B, Smith W et al. Bioanalytical method validation for macromolecules in support of pharmacokinetic studies. Pharm. Res. 22(1), 1425-1431 (2005).

7 European Medicines Agency. Guideline on Bioanalytical Method Validation (2011). www.ema.europa.eu/documents/scientific-guideline/guideline-bioanalytical-method-validation_en.pdf

8 US FDA. Draft Guidance for Industry: Bioanalytical Method Validation (2013).

9 US FDA. Guidance for Industry: Bioanalytical Method Validation (2018). www.fda.gov/downloads/Drugs/Guidances/ucm070107.pdf

10 Findlay J, Dillard R. Appropriate calibration curve fitting in ligand binding assays. AAPS J. 9(2), E260-E267 (2007).

11 Booth B, Arnold ME, DeSilva B et al. Workshop report: Crystal City V - quantitative bioanalytical method validation and implementation: the 2013 Revised FDA Guidance. AAPS J. 17(2), 277-288 (2015).

12 Findlay J, Smith W, Lee J et al. Validation of immunoassays for bioanalysis a pharmaceutical industry perspective. J. Pharm. Biomed. Anal. 21, 1249-1273 (2000).

13 ANVISA. Resolution RDC 27 Minimum Requirements for Bioanalytical Method Validation Used in Studies with the Purpose of Registration and Post-registration of Medicines. Agencia National de Vigilancia Sanitaria, Brazil (2012).

14 Japan, MHLW. Guideline on bioanalytical method validation in pharmaceutical development (2013). www.nihs.go.jp/drug/BMV/250711_BMV-GL.pdf

15 Japan, MHLW. Guideline on bioanalytical method (ligand binding assay) validation in pharmaceutical development (2014). www.nihs.go.jp/drug/BMV/260530_LBA-GL_E.pdf.

16 Compendium of papers from the Global Bioanalysis Consortium. www.globalbioanalysisconsortium.org/GBCPapers

17 European Medicines Agency. Reflection paper for laboratories that perform the analysis or evaluation of clinical trial samples (2012). www.ema.europa.eu/en/documents/regulatory-procedural-guideline/reflection-paper-guidance-laboratories-performanalysis-evaluation-clinical-trial-samples_en.pdf

18 US FDA. Guidance for industry, immunogenicity testing of therapeutic protein products - developing and validating assays for anti-drug antibody detection (2019). www.fda.gov/ucm/groups/fdagov-public/@fdagov-drugs-gen/documents/document/ucm629728.pdf

19 Casadevall N, Nataf J, Viron B et al. Pure red-cell aplasia and antierythropoietin antibodies in patients treated with recombinant erythropoietin. N. Engl. J. Med. 346, 469-475 (2002).

20 Li J, Yang C, Xia Y et al. Thrombocytopenia caused by the development of antibodies to thrombopoietin. Blood 98, 3241-3248 (2001).

21 Chung $\mathrm{CH}$, Mirakhur B, Chan E et al. Cetuximab-induced anaphylaxis and IgE specific for galactose-alpha-1,3-galactose. N. Engl. J. Med. 358, 1109-1117 (2008). 
22 European Medicines Agency, Committee for Medicinal Products for Human Use (CHMP). Guideline on immunogenicity assessment of therapeutic proteins (2017).

www.ema.europa.eu/en/documents/scientific-guideline/guideline-immunogenicity-assessment-therapeutic-proteins-revision-1_en.pdf

23 Piccoli SP. The FDA/C-path/Duke-Margolis public workshop on analytical validation of assays for biomarker qualification: an update. Bioanalysis 10(12), 893-896 (2018).

24 International Council for Harmonisation of Technical Requirements for Pharmaceuticals for Human Use (ICH) M10 Concept Paper. www.ich.org/fileadmin/Public_Web_Site/ICH_Products/Guidelines/Multidisciplinary/M10/ICH_M10_Concept_paper_final_7Oct201 6.pdf 
